402 J. Il berg, De Hippocratis Epidemiorum libri tertii etc.

SERIES POSTERIOR.

$\bar{\alpha}$ ПТФАҮРК $\mathrm{V}, \Pi$ pro $\mathrm{T}$ Littr.

$\bar{\beta} \Pi \triangle \wedge \in \Gamma \Theta \mathrm{V}, \Pi$ pro $\Gamma$ Foesius

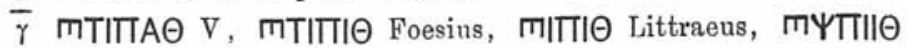
temptavi

$\bar{\delta} m P C \Theta V, m \mid C \Theta$ sive $m \mid Z \Theta$ ex Parisinis Littraeus, $\Phi$ pro $P$ ego

$\bar{\varepsilon}$ mҮ $\triangle \Theta \Pi$ ТАВГ $\triangle \Theta \mathrm{V}$, ex duplici serie conflatas esse litteras perspexi

$\bar{\varsigma} m \triangle I A Y T T A \Theta I B A Y \mathrm{~V}$, alteram seriem non tetigi

$\bar{\zeta}$ pro $\mathrm{O}$ voluit I (iôpús) Darembergius

$\bar{\eta} \Pi$ explicari potest $\pi \tau \dot{\varepsilon} \varepsilon \lambda . \varsigma_{\text {s secundum clavem }}$

i mXSIKAY $V$, correxit Foesius

เั $\Pi M \Gamma I Z \Theta \nabla$, correxit Littraeus. $M$ licet interpretari $\mu \cdot \dot{\tau} \tau \rho \alpha$.

Lipsiae.

Ioannes Ilberg.

\title{
Anthol. X 23. XI 84 .
}

In dem mehr skoptischen als protreptischen Gedicht des Automedon X 23, 5 heißt es von einem Rhetor Niketes

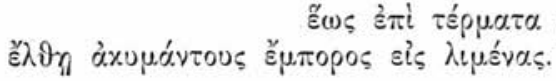

V. 6 ist हैumopos überliefert, vermuthlich falsch; wir brauchen ein Wort, welches die Bedeutung des glücklichen Einfahrens in den Hafen hat; von selbst ergiebt sich daher: $\varepsilon \cup ̌ \pi \lambda_{0} \circ \varsigma$

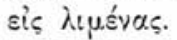

In dem Epigramm des Lucilius XI 84 lesen wir V. 5

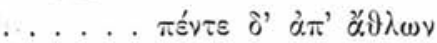

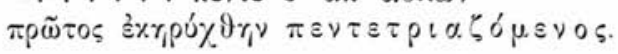

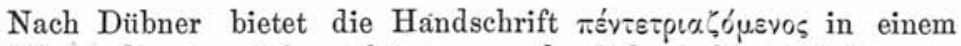
Wort; dies ist nicht richtig, ganz deutlich sind zwei Worte zu erkennen; und diese Lesart wollen wir beibehalten, denn viel besser erklärt sich das doppelte $\pi \varepsilon ́ v \tau \varepsilon$, als daß wir eine so ungeheuerliche Neubildung annehmen.

Göttingen.

P. Salkolowslei. 